\title{
Location of Parathyroid Adenomas in Primary Hyperparathyroidism: Where to Look?
}

${ }^{1}$ Pinar Yazici, ${ }^{2}$ Mehmet Mihmanli, ${ }^{3}$ Emre Bozdag, ${ }^{4}$ Nurcihan Aygun, ${ }^{5}$ Mehmet Uludag

\section{ABSTRACT}

Purpose: Preoperative localization studies for parathyroid adenomas are very essential to perform minimal invasive parathyroidectomy (MIP) with decreased operative time and potential complications. Although most of these studies based on radiological imaging, intraoperative assessment provides the most accurate anatomical description of the location of parathyroid adenomas. In this study, we aim to evaluate the surgical variations of locations of parathyroid adenomas in patients performed parathyroid surgery for primary hyperparathyroidism (PHPT).

Materials and methods: Between January 2010 and December 2013, 243 patients (201 women/42 men) who underwent parathyroid surgery due to PHPT were included. A total of 254 parathyroid adenomas were detected. Demographic features, preoperative work-up, surgical approach, types of procedures and postoperative complications were noted. Locations of parathyroid adenomas were recorded from operative notes. Statistical analysis was performed using t-test and Chi-square. continuous data are expressed as mean \pm standard deviation.

Results: With regard to the most frequently observed, location of adenomas were as follows; right inferior ( $n=89,37.7 \%$ ), left inferior $(n=78,33 \%)$, right superior $(n=44,18.6 \%)$, left superior ( $n=25,10.5 \%$ ) and ectopic locations $(n=18)$. Ectopic adenomas were mostly located in the thymus $(n=9)$ and intrathyroidal tissue $(n=6)$ at a rate of $83 \%$. Postoperative hypocalcemia (11\%) was mostly seen in those with parathyroid adenoma located around the inferior lobes of the thyroid (86\%) and undergoing bilateral neck exploration (75\%).

Conclusion: The most of the parathyroid adenomas were found in orthotopic position and located around the lower pole of the thyroid gland. Ectopic adenomas were mostly located in thymus or intrathyroidal. Postoperative hypocalcemia was also higher in those with parathyroid adenoma located around the inferior lobe of the thyroid.

Keywords: Parathyroid anatomy, Primary hyperparathyroidism, Parathyroid adenoma, Surgical Approach.

How to cite this article: Yazici P, Mihmanli M, Bozdag E, Aygun N, Uludag M. Location of Parathyroid Adenomas in

\footnotetext{
${ }^{1}$ Chief of Staff, ${ }^{2}$ Professor, ${ }^{3,4}$ Resident, ${ }^{5}$ Associate Professor

${ }^{1-5}$ Department of General Surgery, Sisli Etfal Training and Research Hospital, Turkey
}

Corresponding Author: Pinar Yazici, Chief of Staff, Department of General Surgery, Sisli Etfal Training and Research Hospital Turkey, Phone: +90 2123735000, e-mail: drpinaryazici@ gmail.com
Primary Hyperparathyroidism: Where to Look? World J Endoc Surg 2015;7(1):1-5.

Source of support: Nil

Conflict of interest: None

\section{INTRODUCTION}

Parathyroid surgery is quite different from all other surgical procedures. The mentality of the surgery depends on determining diseased parathyroid gland, as others have never even heard. This is why the surgeons face with a challenging course to clarify which one becomes diseased in the preoperative period. To achieve the best outcome, it is essential to be familiar with both embryogenesis of parathyroid glands, which have the most variable anatomy in human body and neck anatomy as well.

Along with advances in minimally invasive surgery, minimally invasive parathyroidectomy (MIP) by the focused mini-incision technique has been gaining popularity and, moreover, it is becoming the standard of care in primary hyperparathyroidism (PHPT) facilitating the same-day surgery. It is very important to clarify the location of the adenomas in the preoperative period. Preoperative diagnostic studies serve to explore the locations of parathyroid adenomas, which can take place within an extensive region between jaw and heart. ${ }^{1,2}$ Despite of technological advances, this considerable variation can lead to failure to excise the right adenomatous parathyroids. Therefore, both experience of the surgeon and information on the most likely locations have a significant role in parathyroid surgery, particularly for ectopically located ones. The ultimate aim is to have a conjecture about the route of surgical procedure avoiding bilateral neck exploration and its drawbacks. ${ }^{3}$ Moreno et al developed a nomenclature system that specifies the locations of parathyroid adenomas and they aimed to improve the predicting accuracy of parathyroid gland location to determine the correct surgical approach. ${ }^{4}$ It includes very detailed information about locations of parathyroid adenoma. However, it is usually difficult to determine locations precisely enough based on preoperative diagnostic work-up.

Although there are some studies showing the locations of parathyroid adenomas ${ }^{4,5}$ mostly regarding primarily the preoperative diagnostic imaging studies, ${ }^{6,7}$ there is 
still no consensus on variations of the locations due to inconsistent and contradictory results. In this study, we focused our attention on two issues: the anatomical distribution of parathyroid adenomas regarding the surgical findings in patients who underwent parathyroid surgery due to PHPT and postoperative outcome with respect to the location of adenoma.

\section{MATERIALS AND METHODS}

Between January 2010 and December 2013, we retrospectively reviewed the records of 243 patients undergoing parathyroidectomy for PHPT. Data collection included demographic variables, symptoms, preoperative diagnostic work-up, surgical approach and treatment modality, additional surgical procedures performed, postoperative complications and histopathological examination. Diagnosis of PHPT was confirmed with laboratory results, which demonstrated increased or inappropriate serum calcium and parathyroid hormone (PTH) levels. Preoperative imaging diagnostic work-up for location of the parathyroid adenomas included neck ultrasonography (US) and/or Tc-99m-sestamibi (MIBI) scans. In those with discordant or negative results for adenoma location, magnetic resonance imaging (MRI) was the next step in the work-up.

Regarding intraoperative observation, adenomatous gland locations were classified according to the routine anatomic location of parathyroid adenomas as follows: right superior (Rs), right inferior (Ri), left superior (Ls), left inferior (Li) and ectopic (E-intrathymic, within thyroid gland, close to clavicles, tracheoesophageal groove and mediastinum). Particularly for superior parathyroid glands, tracheoesophageal groove is defined as ectopic location due to paraesophageal migration of the glands.

The three different types of approaches including Kocher's incision for bilateral exploration, minimal invasive focused lateral approach and anterior approach were used to identify the parathyroid adenomas. An intraoperative $\mathrm{PTH}$ analysis was used to determine operative success and it was indicated by a decrease in PTH greater than $50 \%$ of the preoperative value.

Statistical analyses were performed using SPSS version 20 (Statistical Package for the Social Sciences Inc, Chicago, IL, USA). Differences between continuous and categorical variables were assessed by Student's t-test and Fisher's exact test or Chi-square test, respectively. A value of $p<0.05$ was considered statistically significant.

\section{RESULTS}

We recorded 254 parathyroid adenomas in 243 patients with PHPT. There were female dominance (42 males $/ 201$ females), and the mean age was $55.6 \pm 12.6$ years (29-93).
Only $27 \%$ of patients were diagnosed due to symptoms including bone disease $(n=25)$, weakness and fatigue $(\mathrm{n}=22)$ and nephrolithiasis $(\mathrm{n}=18)$. All patients were performed neck US in which 13 (5.3\%) was negative for parathyroid adenoma and 25 patients $(10 \%)$ were found with suspicious imaging for parathyroid adenomas. Other diagnostic methods included 99mTc-MIBI and MRI of neck for demonstration of abnormal parathyroid gland in $196(80 \%)$ and 55 (22\%) patients, respectively. The sensitivity of US, MIBI and MRI for the detection of abnormal parathyroid gland was 94,84 and $63 \%$ respectively.

In 234 patients $(96 \%)$, single adenoma was isolated, whereas nine cases $(3.7 \%)$ were detected with multiple parathyroid adenomas (two adenomas in 7 and three adenomas in 2 patients) (Fig. 1). Among perithyroidal located 236 parathyroid adenomas, the Ri parathyroid gland was the most common site of adenomas with a rate of $37.7 \%(n=89)$. The other most common location was Li region ( $\mathrm{n}=78,33 \%$ ). The distribution of the remaining adenomas was as follows: 44 on the Rs (18.6\%), 25 on the Ls (10.5\%), and the ectopic adenomas in 18 patients $(7.4 \%$, 18/243) (Fig. 1). Ectopic locations included thymus $(n=9)$, intrathyroidal region $(n=6)$, mediastinum $(n=2)$, and carotid sheath $(\mathrm{n}=1)$. While 15 ectopic adenomas were found as single, three of them were accompanied with others, which were located on perithyroidal regions.

The median size of adenomas was $1.5 \mathrm{~cm}$ [mean: $1.8 \pm$ $0.8 \mathrm{~cm}(0.7-4.9)$ ]. Those located in the inferior region ( $\mathrm{Li}$ and $\mathrm{Ri}$ ) had higher diameter compared to adenomas placed superiorly (Ls and Rs) (1.8 \pm 0.9 vs 1, $7 \pm 0.7)$, but it was statistically insignificant $(\mathrm{p}=0.141)$.

Minimally invasive parathyroidectomy was performed in 126 cases (51.8\%). Of these, 109 patients $(86.5 \%)$ underwent lateral focused approach. Four of them (3\%) were converted to standard bilateral exploration. Bilateral neck exploration through a Kocher's incision was performed in 117 patients (48\%) (Fig. 2). Subtotal parathyroidectomy (3.5 glands) and cervical thymectomy were

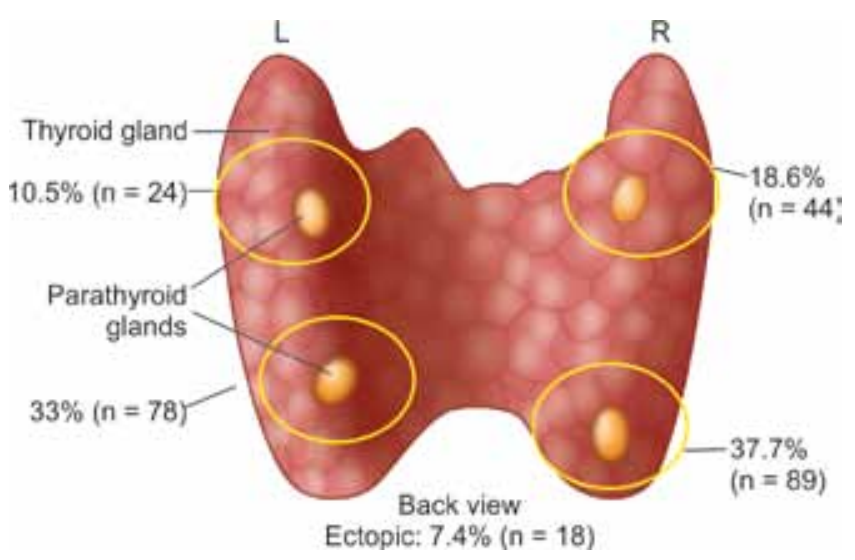

Fig. 1: Surgical variations of locations of the parathyroid adenomas 


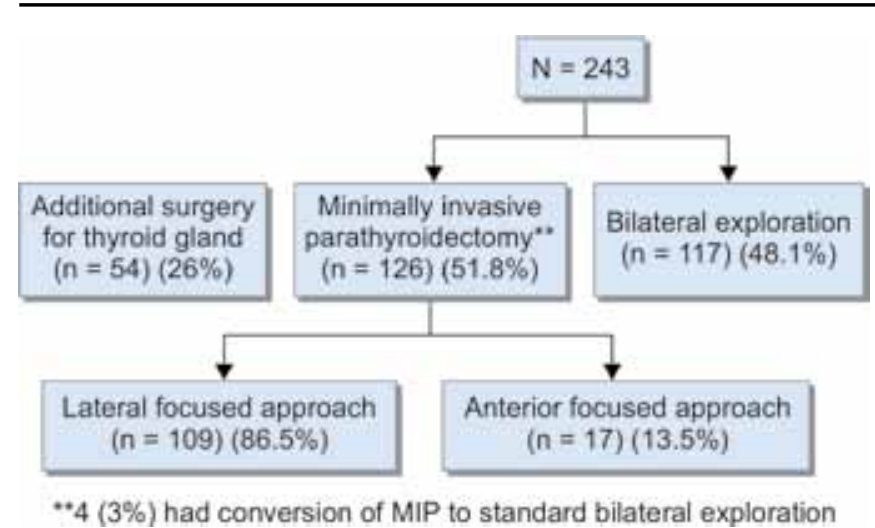

Fig. 2: Types of surgical procedures and approaches to the surgical site

performed in seven patients $(2.8 \%)$ due to undetectable adenoma. The rest parathyroid tissue was marked with a titanium clip.

Additional surgery for thyroid gland was performed in 54 patients $(26.8 \%)$. While papillary carcinoma and microcarcinomas were detected incidentally in one and three patients $(1.6 \%)$ respectively, parathyroid carcinoma was also diagnosed in two patients $(0.8 \%)$. The location of parathyroid carcinoma was inferior region (one Ri and Li).

Overall postoperative hypocalcemia rate was $11 \%$ ( $\mathrm{n}=29)$. Transient hypocalcemia was detected in 24 patients and all of them had parathyroid adenomas placed inferior locations but three $(87.5 \%)(p=0.004)$. The patients with bilateral neck exploration had higher rate of transient postoperative hypocalcemia compared to those with MIP (14.8 vs $4.9 \%$ respectively, $\mathrm{p}=0.011)$. Only five patients were diagnosed with hypocalcemia requiring calcium replacement in the first year after surgery, whereas $97.9 \%$ of patients were normocalcemic. All but one of those with persistent hypocalcemia underwent bilateral neck exploration. Of these patients, three underwent parathyroidectomy with thymectomy.

\section{DISCUSSION}

Apart from the embryogenesis and preoperative diagnostic work-up, knowledge regarding the potential differences in parathyroid gland locations facilitates better surgical planning, as it also depends on the surgical experience. ${ }^{8}$ Most of the data about location of parathyroid adenoma are based on results of preoperative imaging methods. There have not been many studies on the surgical localization of parathyroid adenomas (Table 1). ${ }^{4,5,9}$ Nevertheless, intraoperative assessment provides the most accurate anatomical description of the location of parathyroid adenomas. In this study, we demonstrated the surgically-observed locations of parathyroid adenomas in our series.

Migration of the parathyroid glands during fetal development explains why the surgical approach to the parathyroid glands can get complicated to do. Despite of the same endpoint, detecting the diseased parathyroid gland finally, surgical approaches (minimal invasive technique including lateral focused approach or standard bilateral neck exploration, etc) and some other details about procedures may vary from center to center. The upper parathyroid glands are placed in rather familiar locations; whereas lower parathyroid glands are puzzling due to more complicated migration process. In our series, most of the parathyroid adenomas (70\%) were found around inferior lobes of the thyroid gland, particularly on the Ri in our series. Interestingly, the right side was also common for those adenomas located on the upper pole of thyroid (18 vs 10\%). Likewise, previous surgical-based studies reported the inferior lobes of thyroid gland as the most common location for parathyroid adenomas. ${ }^{4,5,9}$ Marzouki et al also reported similar type of side-predisposition for the left side. ${ }^{5}$

Currently, the diagnostic imaging studies are usually obtained to facilitate MIP avoiding extensive exploration that can cause damage to the other parathyroid glands or the recurrent laryngeal nerve. The accuracy of the parathyroid tumor's localization by US depends on variable parameters including size and location with a sensitivity rate of 70 to $80 \%{ }^{10,11}$ Recent studies emphasized the utility of Tc-99m-MIBI scan in successful MIP with unilateral neck exploration. ${ }^{12,13}$ High-quality scintigraphy with Tc-99m-MIBI can accurately localize parathyroid adenomas in 85 to $95 \%$ of patients with PHPT. ${ }^{14-16}$ However, because close location of parathyroid glands to thyroid gland that is also metabolically active, results may not be satisfactory, particularly for intrathyroidal adenoma. In our study, US was performed in all patients with negative or inconclusive result at a rate of $15 \%$, whereas Tc-99m-MIBI scan and MRI were applied for 80 and $22 \%$ of the patients with an accuracy rate of 84 and $63 \%$, respectively. Detailed explanation about imaging studies were not given because this study was planned based on surgically-observed locations.

Advances in diagnostic methods allow performing focused parathyroid surgery that 126 patients underwent MIP in our series. This approach can be performed

Table 1: Review of the literature regarding adenoma locations reported in surgical studies of primary hyperparathyroidism

\begin{tabular}{lllll}
\hline Years & Study & No. of patients & Superior (\%) & Inferior (\%) \\
\hline 2010 & Marzouki et al & 121 & $23(\mathrm{R}-8.2, \mathrm{~L}-15)$ & $74(\mathrm{R}-33, \mathrm{~L}-41)$ \\
2011 & ${\text { Moreno et } \mathrm{al}^{4}}^{5}$ & 271 & 29.8 & 41.6 \\
2012 & Mazeh et al $^{9}$ & 108 & 47 & 49 \\
\hline
\end{tabular}


through a very small incision under local anesthesia and, therefore, outpatient surgery is available. Besides, complications including recurrent laryngeal nerve paralysis and postoperative hypocalcemia are also lower. ${ }^{17}$ Not surprisingly, in all patients with persistent hypocalcemia, but one, surgical approach was bilateral neck exploration. Moreover, it is also noteworthy that $87 \%$ of the patients who required postoperative calcium replacement had parathyroid adenoma located around the inferior lobes of the thyroid gland. This high incidence can be attributable to risk of injury to parathyroid glands during exploration proportionally increased with the variable locations of lower parathyroid adenomas. At this point, apart from imaging results that sometimes fail to address the exact location, surgeons' experience and knowledge about surgical variations may have major role. Gamma probeguided MIP is also very helpful to determine the location of parathyroid adenoma, particularly those located in an ectopic position; ${ }^{18-20}$ however, it requires technical support from nuclear medicine department and a close working relationship.

Although 80 to $85 \%$ of parathyroid adenomas are found adjacent to the thyroid gland in their normal location, 15 to $20 \%$ are ectopically placed. ${ }^{21}$ Ectopic locations should be considered, such as within or outside of the thyrothymic ligament, in the mediastinum, along the esophagus and within or lateral to the carotid sheath. In our study, $7.4 \%$ of patients with ectopic parathyroid adenoma were detected and thymus was the most common location (50\%). Intrathyroidal location was found with a rate of $2.5 \%$, which was comparable to the incidence of 0.08 to $4 \%$ reported in the literature. ${ }^{4,22-24}$ One of the several challenging issues is multiple-gland adenomas that we detected in nine cases $(3.7 \%)$, consistent with the previous reports. ${ }^{25}$

In conclusion, most of the parathyroid adenomas were found in orthotropic position; around or in proximity of the inferior lobes of thyroid gland, particularly right side. This distribution was also valid for ectopic adenomas that were mostly located in thymus following intrathyroidal location. Likewise, postoperative hypocalcemia was higher for inferiorly localized parathyroid adenomas. We believe that apart from a thorough understanding of the embryologic development of the parathyroid glands and preoperative diagnostic techniques, knowledge about these surgical-based variations may improve surgical success.

\section{REFERENCES}

1. Randolph GW, Urken ML. Surgical management of primary hyperparathyroidism. In: Randolph GW, editor. Surgery of thyroid and parathyroid glands. Philadelphia, Pa: WB Saunders; 2003. p. 507-528.
2. Steward DL, Hairston JA. Development and surgical anatomy of the thyroid compartment. In: Terris DJ, Gourin CG, editors. Thyroid and Parathyroid Diseases. New York, NY: Thieme; 2009. p. 11-17.

3. Debridge L, Younes N, Guinea A, Reeve TS, Clifton-Bligh P, Robinson BG. Surgery for primary hyperparathyroidism 1962-1996: indications and outcome. Med J Aust 1998;168(4): 153-156.

4. Moreno MA, Callender GG, Woodburn K, et al. Common locations of parathyroid adenomas. Ann Surg Oncol 2011; 18(4):1047-1051.

5. Marzouki HZ, Chavannes M, TamiliaM, et al. Location of parathyroid adenomas: 7-year experience. J Otolaryngol Head Neck Surg 2010;39(5):551-554.

6. Civelek AC, Ozalp E, Donovan P, Udelsman R. Prospective evaluation of delayed technetium-99m sestamibi SPECT scintigraphy for preoperative localization of primary hyperparathyroidism. Surg 2002;131(2):149-157.

7. Lumachi F, Zucchetta P, Marzela MC, et al. Advantages of combined technetium-99m-sestamibi scintigraphy and high resolution ultrasonography in parathyroid localization: comparative study in 91 patients with primary hyperparathyroidism. Eur J Endocrinol 2000;143(6):755-760.

8. Christenson T, Hellstrom K, Wengle B, Alveryd A, Wikland B. Prevalence of hypercalcemia in a health screening in Stockholm. Acta Med Scand 1976;200(1-2):131-137.

9. Mazeh H, Stoll SJ, Robbins JB, Sippel RS, Chen H. Validation of the Perrier parathyroid adenoma location nomenclature. World J Surg 2012;36(3):612-616.

10. Neumann DR, Esselstyn CB, Go RT, Wong CO, Rice TW, Obuchowsky NA. Comparison of double-phase 99mTcsestamibi with 123I-99mTc-sestamibi subtraction SPECT in hyperparathyroidism. AJR 1997;169(6):1671-1674.

11. Francis IS, Loney EL, Buscombe JR, Thakrar DS, Berger L, Hilson AJW. Technetium-99m-sestamibi dual-phase SPECT imaging: concordance with ultrasound. Nucl Med Commun 1999;20(2):487-488.

12. Rubello D, Piotto A, Casara D, Muzzio PC, Shapiro B, Pelizzo MR. Role of gamma probes in performing minimally invasive parathyroidectomy in patients with primary hyperparathyroidism: optimization of preoperative and intraoperative procedures. Eur J Endocrinol 2003;149(1):7-15.

13. Karyagar S, Karyagar SS, Yalcin O, Yuney E, Mulazimoglu M, Ozpacaci T, et al. Gamma probe guided minimally invasive parathyroidectomy without quick parathyroid hormone measurement in the cases of solitary parathyroid adenomas. Mol Imaging Radionucl Ther 2013;22(1):3-7.

14. Chen H, Sokoll LJ, Udelsman R. Outpatient minimally invasive parathyroidectomy: a combination of sestamibi-SPECT localization, cervical block anesthesia, and intraoperative parathyroid hormone assay. Surg 1999;126(6):1016-1021.

15. Kumar A, Cozens NJA, Nash JR. Sestamibi scan-directed unilateral neck exploration for primary hyperparathyroidism due to a solitary adenoma. Eur J Surg Oncol 2000;26(8):785-788.

16. Stephen AE, Roth SI, Fardo DW, et al. Predictors of an accurate preoperative sestamibi scan for single-gland parathyroid adenomas. Arch Surg 2007;142(4):381-386.

17. Lew JI, Rivera M, Irvin GL, Solorzano CC. Operative failure in the era of focused parathyroidectomy: a contemporary series of 845 patients. Arch Surg 2010;145(7):628-633.

18. Flynn MB, Bumpous JM, Schill K, McMasters KM. Minimally invasive radioguided parathyroidectomy. J Am Coll Surg 2000;191(1):24-31. 
19. Norman J, Cheda H. Minimally invasive radioguided parathyroidectomy facilitated by intraoperative nuclear mapping. Surg 1997;122(6):998-1004.

20. Grant CS, Thompson G, Farley D, van Heerden J. Primary hyperparathyroidism surgical management since the introduction of minimally invasive parathyroidectomy: mayo clinic experience. Arch Surg 2005;140(5):472-478.

21. Feliciano DV. Parathyroid pathology in an intrathyroidal position. Am J Surg 1992;164(5):496-500.

22. Phitayakorn R, McHenry CR. Incidence and location of ectopic abnormal parathyroid glands. Am J Surg 2006;191(3):418-423.
23. Geatti O, Shapiro B, Orsolon P, et al. Localization of parathyroid enlargement: experience with technetium $99 \mathrm{~m}$ methoxyisobutylisonitrile and thallium-201 scintigraphy, ultrasound and computed tomography. Eur J Nucl Med 1994; 21(1):17-23.

24. Carty SE, Worsey MJ, Virji MA, Brown ML, Watson CG. Concise parathyroidectomy: the impact of preoperative SPECT 99mTc sestamibi scanning and intraoperative quick parathormone assay. Surgery 1997;122(6):1107-1116.

25. Tezelman S, Shen W, Shaver JK, et al. Double parathyroid adenomas. Clinical and biochemical characteristics before and after parathyroidectomy. Ann Surg 1993;218(3):300-307. 